\title{
Article \\ Proximity, Familiarity or Congruency? What Influences Memory of Brand Placement in Videogames
}

\author{
Irene Aliagas ${ }^{1, * \mathbb{D}}$, Jesús Privado ${ }^{2}$ and M Dolores Merino ${ }^{1}$ \\ 1 Facultad de Psicología, Campus de Somosaguas, Universidad Complutense de Madrid, Pozuelo de Alarcón, \\ 28223 Madrid, Spain; lolamerino@psi.ucm.es \\ 2 División de Psicología, Centro de Enseñanza Superior Cardenal Cisneros, 28006 Madrid, Spain; \\ jesus.privado@pdi.ucm.es \\ * Correspondence: irealiag@ucm.es
}

check for

updates

Citation: Aliagas, I.; Privado, J.; Merino, M.D. Proximity, Familiarity or Congruency? What Influences Memory of Brand Placement in Videogames. J. Theor. Appl. Electron. Commer. Res. 2021, 16, 811-827. https://doi.org/10.3390/jtaer16040046

Received: 17 December 2020

Accepted: 24 January 2021

Published: 30 January 2021

Publisher's Note: MDPI stays neutral with regard to jurisdictional claims in published maps and institutional affiliations.

Copyright: (c) 2021 by the authors. Licensee MDPI, Basel, Switzerland. This article is an open access article distributed under the terms and conditions of the Creative Commons Attribution (CC BY) license (https:// creativecommons.org/licenses/by/ $4.0 /)$.

\begin{abstract}
Brand placement in videogames consists in integrating various brands that are not intrusive to give a feeling of reality to the context, as such increasing awareness and recognition of the brand in the mind of the consumer. The goal of this study is to ascertain if brand position, familiarity and congruency influence memorization of brands presented in a racing videogame. An experimental design 2 (position: prominent or subtle) $\times 2$ (congruency: yes or no) $\times 2$ (familiar: yes or no) was used in a sample of 117 participants $(M=20.91$ years, $S D=1.75$ years). Consumers have better recall and recognition of brand placement when it is familiar. Likewise, there is more memorization of placement that is congruent with the videogame's subject matter. Nevertheless, it appears that position has no influence on memory. Regarding the three-way interaction, it turns out that the best way to remember brands is when they are familiar to the consumers, congruent with the videogame's genre, and they are positioned in a prominent position. This article is the first to investigate the interaction between the three main variables that affect the memorization of brand placement.
\end{abstract}

Keywords: product placement; brand placement; in-game advertising; brand recognition; brand recall; brand memory

\section{Introduction}

Brand placement occurs when one or several brands are inserted into movies, television series, videogames, etc., to make the context seem more realistic, but without being intrusive. The object is for brand awareness and recognition to be increased in the mind of the consumer [1,2]. This practice began in the 1980s and has been used in a multitude of media: cinema $[3,4]$, TV [5,6], music $[7,8]$ and online video platforms $[9,10]$. One of the most relevant of these examples is videogames, given that this is a market in full development $[1,11,12]$ whose level of worldwide revenue was $\$ 137.9$ billion in 2018 [13].

Focusing on the videogame industry, there are two ways to use brand placement. The first is called ad-game or advergame, where the brand takes a leading role in the game; thus, the avatar the game is played with would be the brand itself. For example, the Android lollipop version offers a free game in which the Android doll has to dodge the lollipops that appear on the scene. The second is in-game advertising, where the game is the main thing and the brands appear in the background [14,15], for example, advertising banners that often appear in shooting or sporting games.

In comparison with other devices or channels, videogames allow greater exposure time for the brand and more interactivity with it. In fact, whenever the video game is accessed, the brand placement (BP) will be present and cannot be evaded because it is part of the scene $[5,16]$. This is affirmed since it is not the same passively watching a program or series, in which the BP appears to be carrying out an active task than playing, where all attentional and cognitive resources are oriented to the game. On the other hand, in media such as TV, someone may be performing a task with the program in the background, so 
he or she may not be exposed to BP. In fact, watching a game is not the same as playing it. Wang and Yao [17] found that a higher proportion of BP was remembered and recognized when a virtual reality car game was played than when it was watched. That is why marketers have been so interested in the use of video games as a medium in which to present commercial brands.

Concretely, this study tries to broaden the knowledge about how BP presentation factors influence memorization. For this, the most accepted model within the area is presented, as well as previous literature that helps to clarify the effectiveness of BP in video games, especially in-game advertising. For the first time, the interaction between the three main presentation variables of the BP is jointly analyzed: its position, its congruence with the game and its familiarity. Furthermore, an attempt is made to clarify the role of congruency in memorizing $\mathrm{BP}$, an aspect where contradictory results have been found.

Regarding the structure, in the second section the theoretical framework is presented and the hypotheses to be investigated are discussed. The third section describes the methodology followed. The fourth section presents the results. The fifth section includes discussion, conclusions and main implications of the study. Finally, in the last section, limitations and future lines to be investigated are indicated.

\section{Conceptual Framework and Literature Review}

Based on the model of Balasubramanian, Karrh and Patwardhan [18] or the BKP model, it is assumed that there are BP design factors and other individual factors that influence its processing and that can generate cognitive responses, affective responses and/or conative responses related to the brand presented. Nowadays, this conceptual framework continues to be the most accepted within the BP area [19-22] and that is why it has been chosen as the basis for this study.

The most relevant part of this model is memorization of the BP. This paper investigates the role of the three main design variables of the BP that could influence its recall and recognition: placement in the scene $[23,24]$, the familiarity of the positioned brands [25,26], and its congruence with the videogame [24,27].

\subsection{Brand Placement Position and Memory}

Playing videogames is a task that demands cognitive resources of encoding, storage and retrieval of the stimuli presented. Ultimately, this has to do with aspects involved in memory. Memory is understood to be the conscious recall of information that has recently been presented [28]. Consequently, if while a person plays a video-game they pay attention to brand placement and encode it, it would be possible to access this conscious memory by directly asking the participants what they remember of the stimuli presented $[14,29,30]$.

The traditional tests to measure memory have been recall and recognition. The difference between the two is that recall is a process of searching for information in the memory after having had a learning event, while recognition is not. In recognition, the stimulus is presented and it is only necessary to retrieve its representation from the memory [31].

Videogames demand two tasks during in-game advertising: playing and paying attention to the brand placement inserted in the game. Thus, the limited attention capacity model from Kahneman [32] notes that in tasks that require multiple attentional resources (such as video games) we tend to focus on a main task, which is the one that demands more cognitive resources, giving less attentional resources to the other cognitive processes the game might require (such as the processing of brand placement). This idea is in line with Lull et al. [29], who presented brands on billboards in two types of game, one violent and the other non-violent. In the end, those who played the non-violent game recalled and recognized more brands because the demand for attentional resources was lower. This form of brand placement on billboards has also been used in car racing games. Chaney et al. [33] analyzed the effectiveness of presenting brands on billboards in subtle or peripheral positions within the game, with players confirming that they had seen the 
billboards but could not remember the brands shown. Conversely, Gangadharbatla [34] wanted to see the differences in brand recall, with brands also placed on billboards, while watching a video game, as opposed to when playing it, finding that recall in the passive task, i.e., watching a recording of the video game, was four times higher than when the game was played (active task).

In regard to brands located in a prominent or central position, Nelson [11] found that participants, as soon as they stopped playing, were able to name 30\% of the brands shown, and those located in the foreground had higher recovery rates. Mau et al. [15] point out that those placed in a prominent shot are more likely to create higher brand recall, while subtle placements are more inconspicuous and players may notice them less. Along these lines, Jeong and Biocca [35] used a shooting game where, when someone was killed, the brand logo appeared in the center of the screen and blood spilled out of it. They also put posters, in peripheral shots, with positioned brands, as players went from one battle site to another. They found that brands that had been in a prominent position were recognized much more quickly. Williams [36] also analyzed whether the position influenced its memorization. He employed a racing game and, after two weeks of playing the game, participants completed a questionnaire indicating which brands they recognized having been present in the video game. They found greater recognition of prominently featured brands (33\%) than subtle ones $(9 \%)$. This would indicate that the player's attention tends to be on the center of the game scene, and by placing a brand in this focal point the player is able to integrate the brand into their gaming experience, that is, into the main task, and as such would devote more processing resources to the brand [25]. In fact, this coincides with the spotlight model of attention, which likens the focus of attention to a spotlight. By this, all the information that is "illuminated" within this focus is processed, while what is outside this area will not be [37].

In short, a prominent position is expected to lead to greater recall and recognition of the BP integrated into the video game.

\subsection{Brand Placement Familiarity and Memory}

Another variable that can affect recall and recognition of brand placement is familiarity with the brand, i.e., all the previous experiences the consumer has had with a specific brand. This ranges from direct experience, such as using a brand's product or service because it was purchased, or indirect experience, such as being exposed to brands because they spontaneously appear around us [26]. Purchase decisions by consumers are influenced by the familiarity they have with the brand [38]. Furthermore, previous research has also indicated that, when they are familiar, it appears that much more information is stored in the memory than with unknown brands, making it possible to retrieve from the memory and use when making a decision $[25,26,39]$. In regard to familiarity, the majority of the research has used subtle positions to present brands, generally resulting in greater recall of familiar brands than unfamiliar ones. Moreover, recall of these brands was worse than recognition. This can be interpreted in that we have much more stored information about known brands, so it is easier for us to remember familiar ones [15,25,26,34,35]. That said, videogames are characterized by a much higher level of brand interaction, and it has been seen that this increases the relevance and recall of the brand when it is familiar [40].

Therefore, it seems that there is greater recall and recognition when the BP presented in the video game is familiar.

\subsection{Brand Placement Congruency and Memory}

Congruence is defined as how the brands presented in the videogame into with the game's genre [41], an aspect that can influence memory of brand placement. Lewis and Porter [42] suggest that advertising inserted in a game must be real and consistent with the game so as not to be rejected, so ideally brands should be used that make sense within the game's subject. Williams [36] studied whether integration and interruption of a BP influenced its memorization. He found greater recognition of those brands congruent 
with the video game genre (15\% automotive brands) than those incongruous ( $8 \%$ luxury brands). Besides, game-interrupting BPs ( $8 \%$ billboards) were less remembered, while more was remembered for integrated BPs (15\% landmarks). On the other hand, although Peters and Leshner [24] did not find interaction effects between proximity and congruency, they did note that their results suggest that there will be greater recognition when the brand is congruent. Nevertheless, there are authors who have found that brands that are incongruent with the videogame have had higher rates of recall and recognition, perhaps as this is unexpected and, therefore, attracts more attention [27]. Hwang et al. [25] showed brands related to sports (congruent) and others that were unrelated (incongruent) on banners within a game of tennis. They found higher rates of recognition of the incongruent brands. Nevertheless, other researchers have seen that there is a higher rate of brand placement recall when there is high congruence with the game, but this also happens when congruence is low [43]. Moreover, these studies have only focused on subtle brand placement.

Thus, it seems that it is unclear whether the presence of congruent BP would lead to greater memory and recognition after playing the video game than incongruous BP.

\subsection{Interaction between Position, Familiarity and Congruence in Brand Memory}

Previous studies have analyzed separately or in pairs the variables in presentation of the BP (position, familiarity and congruence). Since it has not been found that previous studies analyzed the three variables together, this would be a new contribution to the subject investigated. The three appear together within the video game, thus some could influence others and lead to different results in memorization. Hence, the main objective is to find out if the interaction of the three variables leads to greater brand recall and recognition in videogames.

Based on previous literature, the following hypothesis is proposed:

Hypothesis 1 (H1). If brand placement appears in a prominent position, it is familiar, and incongruent with the videogame, then it will be recalled and recognized more than under any other condition.

It has been hypothesized that incongruous BP will achieve greater memorization due to the fact that surprising stimuli are more attended to, and therefore better recalled and recognized, than expected stimuli [44].

\section{Materials and Methods}

In order to respond to the hypotheses, experimental methodology with a $2 \times 2 \times 2$ design was used. This section explains first the preliminary survey used to obtain the stimuli. Then information about participants, devices, stimuli and videogame genre is detailed. In addition, the design, procedure and analyses are described.

\subsection{Preliminary Survey}

Given that the brands would be placed in an automobile videogame in the main study, a questionnaire was developed to identify those brands in accordance with the criteria of familiarity (knowledge and/or experience with the brand) vs. unfamiliarity, and congruence (car brand) vs. non-congruence (laundry detergent brand). These two consumption categories were chosen as, in order not to reject brand placement, it is better if it is real and makes sense within the videogame's subject [42]. A total of 162 Psychology students participated, of which 130 were women $(81 \%)$ and 32 men $(19 \%)$, with an average age of 21 ( $\mathrm{SD}=2.83$ years). The inclusion criterion was to be between 18 and 24 years of age, given that this is the age range that was accessible. All of them reported having been players. Additionally, none of them participated in the main study.

They were shown a total of 40 brands with their logos, 20 for each category. In regard to each brand shown, they had to answer three questions using a Likert-type scale having 
5 points (1: none; 5: much). The first two questions were about familiarity with the brand: "Do you know the brand?", "Have you had any experience with the brand? $[45,46]$ ( $\alpha$ automobile brands $=0.903$; $\alpha$ laundry detergent brands $=0.881$ ); while the third was to measure how much the brand was liked: "Do you like the brand?" [47] ( $\alpha$ automobile brands $=0.853$; $\alpha$ laundry detergent brands $=0.941$ ). Through an online link, participants signed an informed consent and proceeded to fill out the questionnaire, which lasted approximately $15 \mathrm{~min}$.

The mean and standard deviation for familiarity and level of being liked was calculated for each brand. This was done using an Excel program. Then the brands were arranged from highest to lowest, according to preference, thus obtaining a ranking for the car category and for the laundry detergents. For the car category (see Table 1), the most familiar and most liked was BMW (familiarity: $\mathrm{M}=2.54, \mathrm{SD}=0.94$; liked $\mathrm{M}=2.70$, $\mathrm{SD}=1.07$ ) and the least Mahindra (familiarity: $\mathrm{M}=0.06, \mathrm{SD}=0.24$; liked: $\mathrm{M}=1.12$, $\mathrm{SD}=10.97$ ). In contrast, with respect to laundry detergent brands (see Table 2), Ariel was the highest valued (familiarity: $\mathrm{M}=2.95, \mathrm{SD}=0.80$; liked: $\mathrm{M}=2.66, \mathrm{SD}=0.78$ ) and $\mathrm{La}$ Oca the lowest valued (familiarity: $\mathrm{M}=0.06, \mathrm{SD}=0.32$; liked: $\mathrm{M}=1.18, \mathrm{SD}=0.98$ ). Both the familiar brands (BMW and Ariel) as well as the unfamiliar ones (Mahindra and La Oca) had very similar scores, even though they belong to different categories (cars vs. laundry detergents). This means that BMW and Ariel are indistinct or are treated as equals according to familiarity criteria, and the same happens with Mahindra and La Oca with respect to the criteria of unfamiliarity.

Table 1. Descriptive statistics on familiarity and level of being liked for each brand from the automobile sector.

\begin{tabular}{ccc}
\hline Automobile Brand & $\begin{array}{c}\text { Familiarity } \\
\text { Mean (SD) }\end{array}$ & $\begin{array}{c}\text { Liked } \\
\text { Mean (SD) }\end{array}$ \\
\hline BMW & $2.54(0.94)$ & $2.70(1.07)$ \\
Audi & $2.41(0.98)$ & $2.68(0.95)$ \\
Mercedes Benz & $2.36(0.92)$ & $2.57(0.88)$ \\
Ford & $2.50(1.00)$ & $2.18(0.90)$ \\
Renault & $2.46(1.07)$ & $1.92(1.02)$ \\
Peugeot & $2.33(1.07)$ & $1.91(1.05)$ \\
Alfa Romeo & $1.59(0.95)$ & $1.97(1.03)$ \\
Hyundai & $1.80(1.02)$ & $1.73(0.92)$ \\
Lexus & $1.30(1.00)$ & $1.95(1.07)$ \\
Mazda & $1.44(1.01)$ & $1.78(0.97)$ \\
Suzuki & $1.57(0.93)$ & $1.57(0.90)$ \\
Mitsubishi & $1.45(0.99)$ & $1.67(0.91)$ \\
Dacia & $1.49(1.01)$ & $1.52(0.94)$ \\
Infiniti & $0.70(0.98)$ & $1.59(1.11)$ \\
Subaru & $0.73(0.84)$ & $1.51(1.03)$ \\
SangYong & $0.73(0.94)$ & $1.36(0.99)$ \\
Daihatsu & $0.19(0.48)$ & $1.17(0.95)$ \\
Isuzu & $0.10(0.35)$ & $1.19(0.97)$ \\
Oldsmobile & $0.09(0.32)$ & $1.16(0.97)$ \\
Mahindra & $0.06(0.24)$ & $1.12(0.97)$ \\
\hline
\end{tabular}


Table 2. Descriptive statistics on familiarity and level of being liked for each brand from the laundry detergent sector.

\begin{tabular}{ccc}
\hline Laundry Detergent Brand & $\begin{array}{c}\text { Familiarity } \\
\text { Mean (SD) }\end{array}$ & $\begin{array}{c}\text { Liked } \\
\text { Mean (SD) }\end{array}$ \\
\hline Ariel & $2.95(0.80)$ & $2.66(0.78)$ \\
Skip & $2.48(0.92)$ & $2.37(0.85)$ \\
Colon & $2.49(1.00)$ & $2.28(0.95)$ \\
Norit & $2.37(1.11)$ & $2.38(0.98)$ \\
Wipp & $2.43(0.99)$ & $2.18(1.00)$ \\
Dixan & $1.85(0.98)$ & $1.93(0.88)$ \\
Micolor & $1.77(1.14)$ & $1.69(1.01)$ \\
Frosch & $0.36(0.85)$ & $1.39(1.07)$ \\
Mistolin & $0.33(0.74)$ & $1.36(0.97)$ \\
Ekosan & $0.27(0.69)$ & $1.29(0.95)$ \\
Vide & $0.23(0.58)$ & $1.28(0.97)$ \\
Woolite & $0.23(0.69)$ & $1.28(1.02)$ \\
Savia & $0.20(0.55)$ & $1.31(0.95)$ \\
Persil & $0.22(0.60)$ & $1.28(0.96)$ \\
Kiriko & $0.21(0.56)$ & $1.26(0.95)$ \\
Gain & $0.13(0.49)$ & $1.2(0.96)$ \\
Arama & $0.10(0.43)$ & $1.22(0.99)$ \\
Asevi & $0.15(0.49)$ & $1.17(0.94)$ \\
Blanca Nieves & $0.06(0.38)$ & $1.18(0.99)$ \\
La Oca & $0.06(0.32)$ & $1.18(0.98)$ \\
\hline
\end{tabular}

\subsection{Participants}

Efforts were made to ensure that the participants were as homogeneous as possible in order to avoid the presence of potentially contaminating variables, in terms of gender, age, educational level or interest in videogames. Thus, in all experimental conditions there was the same percentage of male and female participants. They had to be between the ages of 18 and 24 because this is the age range that spends the most time playing [48]. The sample was made up of university students, given that $52 \%$ of the players usually belong to this group [48], and all of them must have played videogames. Finally, they must not have or have had any episode or history of any major psychiatric disorder or any illness that could affect proper functioning while completing the task.

A total of 117 students participated, of which 84 were women $(71.8 \%)$ and 33 men $(28.2 \%)$, with an average age of 20.91 years $(\mathrm{SD}=1.75)$. All claimed to be healthy, to have played videogames at some point in their life and to do so now for at least seven hours per week, regardless of the game genre or device used.

\subsection{Devices}

The videogame was presented on a laptop (OS: Windows 10, CPU: Intel ${ }^{\circledR}$ CoreTM i5-8250U @1.60 GHz with Turbo Boost up to 3.4 GHz, RAM: 8 GB with DDR4 memory, Graphics: Nvidia ${ }^{\circledR}$ GeForce ${ }^{\circledR}$ MX150 with 2 GB VRAM) and a 15-inch screen, placing participants at a distance of $40 \mathrm{~cm}$ (15.75 inches).

\subsection{Stimuli and Video Game}

Stimuli. As mentioned in the preliminary survey, the congruent brands belonged to the car category, where BMW was the familiar and Mahindra the unfamiliar. Instead, laundry detergent brands constituted the incongruous category, where Ariel was the familiar and La Oca the unfamiliar.

Video game. Car racing or racetrack games are some of the most commonly utilized for brand placement $[12,45,49,50]$, which is why it was the videogame genre chosen. In addition, reports on the video game industry indicate that, for the age range chosen in the study (18-24 years), $49 \%$ of gamers play driving games [48]. Furthermore, $45.2 \%$ of the participants in the total sample currently play racing games. Four video games 
were created, identical in implementation and design, unlike the product placement and elements that were inserted in them, thus reflecting the different experimental conditions (see Figure 1). To do this, a version of a simple racing game from Unreal Engine Platform was modified.

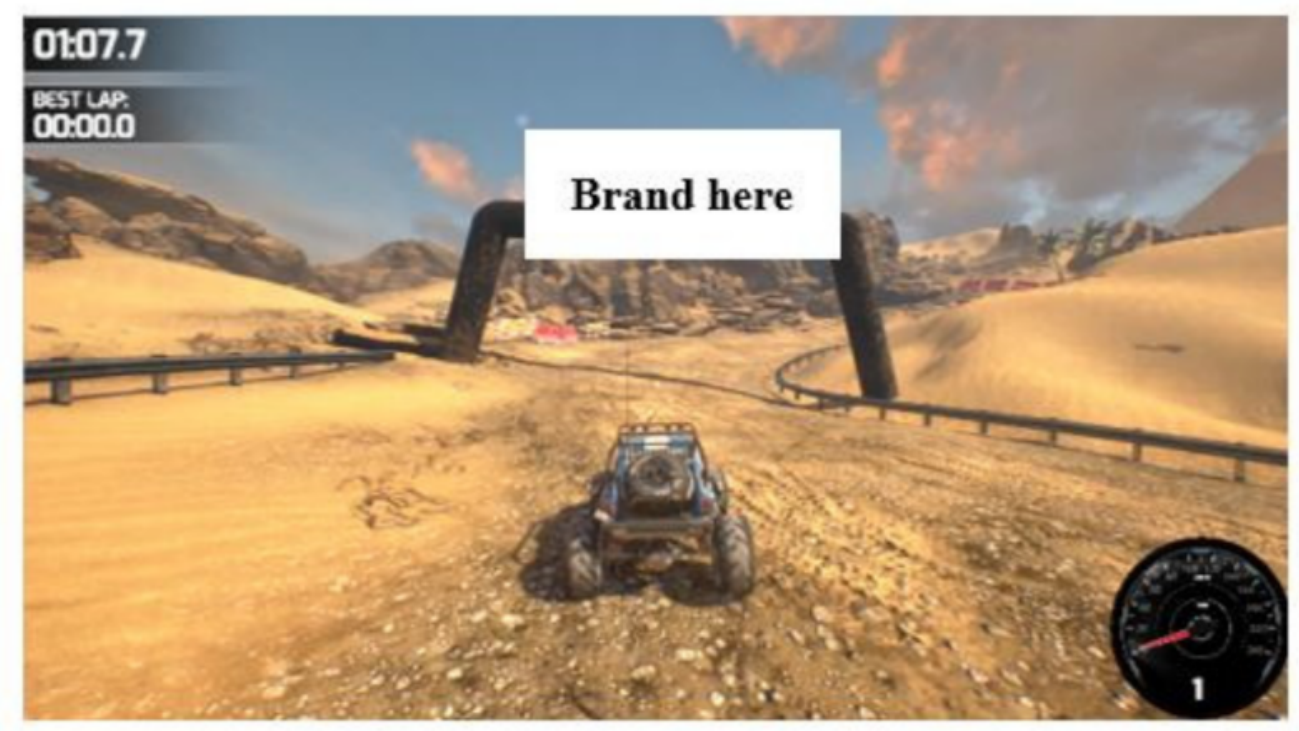

(a) Prominent position

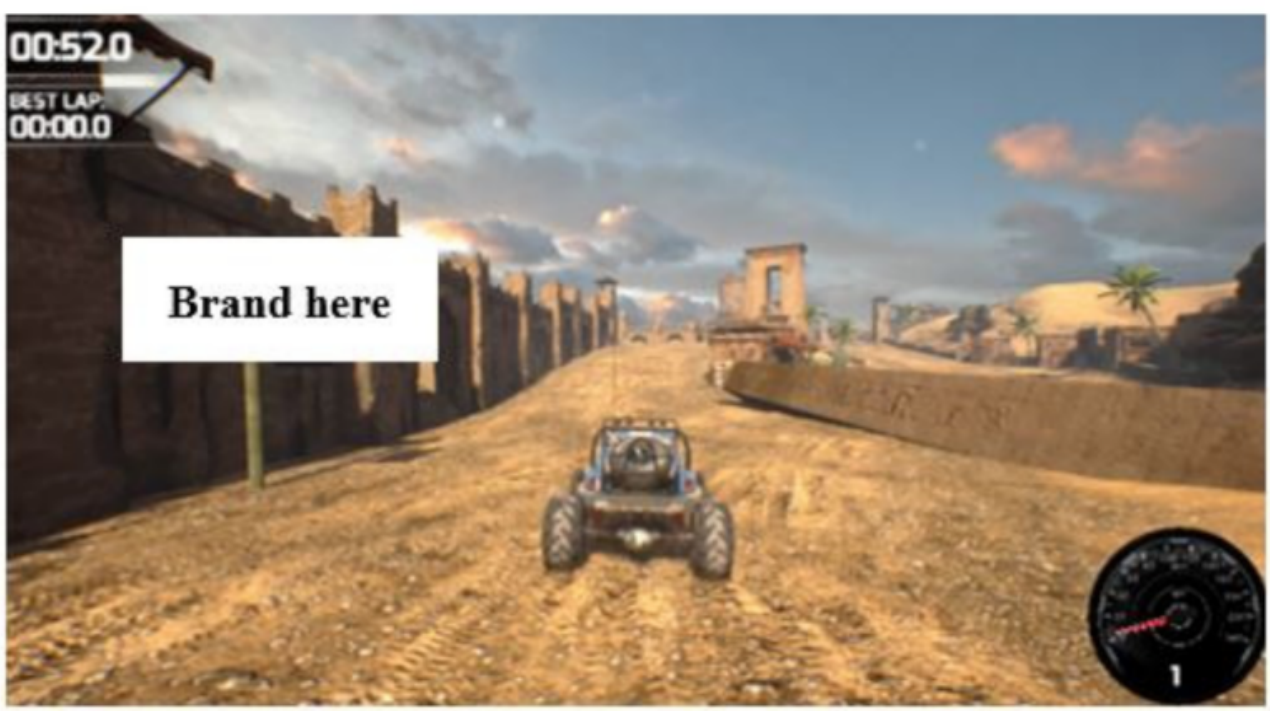

(b) Subtle position

Figure 1. Videogame used in the main study. Examples of brand placements: (a) prominent position, (b) subtle position.

\subsection{Design}

An experimental study was used in which the independent variables were the position of the brand in the game (prominent vs. subtle); familiarity with the brand (familiar vs. unfamiliar); and congruence with the videogame (congruent vs. incongruous). This led to a $2 \times 2 \times 2$ design whose distribution by group can be seen in Table 3. Based on results from the preliminary study, BMW and Ariel were chosen as familiar brands, and Mahindra and La Oca as unfamiliar ones. In terms of congruency, only the familiar brands can be used (BMW for congruent and Ariel for incongruent) because, if there is no brand awareness, whether 
the brand is congruent with the videogame or not cannot be known. The brand position and congruence were between-subject factors and familiarity was a within-subject factor.

Table 3. Itemization of experimental conditions.

\begin{tabular}{|c|c|c|c|c|}
\hline Groups & $\begin{array}{l}\text { Brand Placement (BP) Proximity } \\
\text { (Between-Subject Factor) }\end{array}$ & $\begin{array}{c}\text { BP Congruence } \\
\text { (Between-Subject Factor) }\end{array}$ & $\begin{array}{c}\text { Brand Familiarity } \\
\text { (Within-Subject Factor) }\end{array}$ & $\mathbf{N}$ \\
\hline 1 & Prominent & Congruent & BMW \& Mahindra & 30 \\
\hline 2 & Subtle & Congruent & BMW \& Mahindra & 29 \\
\hline 3 & Prominent & Incongruent & Ariel \& La Oca & 30 \\
\hline 4 & Subtle & Incongruent & Ariel \& La Oca & 28 \\
\hline Total & & & & 117 \\
\hline
\end{tabular}

The dependent variables were recall and recognition of the brand in the videogame.

Due to the existence of certain variables that can affect how cognitive resources are allocated while playing, a series of potentially contaminating variables were analyzed in order to control them and ensure that there were no differences between the different groups. These were:

Previous experience with the game. Participants were asked if they had ever played videogames. Thus, it could be assured that $100 \%$ of the sample had played at some time, therefore not giving statistically significant differences between the different experimental groups $(\mathrm{F} 3,113=2.10, p=0.104, \eta 2$ partial $=0.053)$. The effect size is medium-low ( $\eta 2$ partial $)$. The effect size ( $\eta 2$ partial) according to Cohen's [51] criteria is low for 0.01, medium for 0.06 and large for 0.14 .

Frequency of gaming. Participants were asked to indicate, in minutes, how often they used videogames per week. An average was obtained of $409 \mathrm{~min}$ or $7 \mathrm{~h}$ per week $(\mathrm{SD}=954 \mathrm{~min}$ ) with mesokurtic (kurtosis index $=68.60)$ and positive asymmetric distribution (asymmetry index $=23.92$ ). This is in line with the average gaming time spent by the Spanish population on videogames [52], therefore, the sample is representative in this variable. In addition, there were no statistically significant differences between the groups of participants $(\mathrm{F} 3,113=0.84, p=0.474, \eta 2$ partial $=0.022)$. The effect size is low.

Videogame. Participants were asked whether they had ever played a videogame where they had to drive or go around a racetrack to complete a mission, and $87.18 \%$ confirmed having done this.

\subsection{Procedure}

The study was conducted in a single individual session, with the researcher in charge of giving instructions to the participants, thus ensuring their understanding. First, the participants completed a questionnaire with sociodemographic data, information on whether they had ever played videogames, and confirmation of their experience with videogames involving driving or travelling around racetracks, and their weekly playing time.

Then, participants were randomly assigned to one of the four experimental conditions. In order to control the presence of potentially contaminating variables, the groups were formed with the same proportion of participants in relation to gender. In addition, all had the same educational level, interest in playing videogames and belonged to the age range 18-24 years. Therefore, an experimental design was used to block the effect of these variables.

The next step was to play the videogame. There was no need for any prior training with the task as the videogame controls were very simple. The PC keyboard was used to play, with an option to choose between playing with the arrows on the keyboard or the keys (W, A, S, D), using only one hand. Additionally, subjects had a headset to listen to the game's sounds and not be distracted by other environmental stimuli. The game's topic was a tsunami that had swept away the area of Buqbuq and they needed to save the remaining survivors. This was done by circling the racetrack as fast as possible, and 
after each lap they were shown the number of people they had saved, which came up randomly. These aspects were incorporated into the design: (1) the game's setting, which simulated a desert with sand and palm trees, (2) the appearance of a single vehicle and (3) the impossibility of entering a command so that during the lap they were able to "pick up" survivors. Likewise, participants had to go around the track for four minutes and were shown two brands belonging to the same category (car or detergent) and in the same position (center or periphery) on each of the four laps.

Finally, they answered another questionnaire about their recall and recognition of the brands placed in the game. The session lasted approximately $25 \mathrm{~min}$.

\subsection{Data Analysis}

Two mixed ANOVAs on two between-subject factors were performed: position of the brand in the videogame (prominent or subtle) and congruency (BMW vs. Mahindra and Ariel vs. La Oca) and a within-subject factor: familiar brand (BMW and Ariel) vs. unfamiliar (Mahindra and La Oca). In one ANOVA, brand recall was used as a dependent variable and in the other recognition was used. The analyses were done with the SPSS V. 18 statistical package.

\section{Results}

Table 4 shows the results from the ANOVA tests for recall and recognition. The main results are summarized below, omitting the statistical data shown in the table. In regard to ANOVA assumptions, the covariance matrices are equal for recall but not for recognition, as can be seen from the Box test and variances, which are different for all the groups, formed by the combinations of between-subject factors in line with the results from the Levene test. In any case, it should be taken into consideration that ANOVA is a very robust test when facing a failure to meet these assumptions when group sizes are similar, as in the present study. Moreover, given the study's high N (117), it is to be expected that the hypothesis is rejected [53].

Table 4. Results of mixed ANOVA for recall and recognition based on the different experimental conditions.

\begin{tabular}{|c|c|c|}
\hline Measurement & & Within-Subject Effect \\
\hline \multirow{10}{*}{ Recall } & Familiarity & $\mathrm{F} 1,113=18.84, p<0.001, \eta 2$ partial $=0.143$ \\
\hline & Familiarity Position & $\mathrm{F} 1,113=2.89, p=0.092, \eta 2$ partial $=0.025$ \\
\hline & Familiarity Congruency & $\mathrm{F} 1,113=7.61, p=0.007, \eta 2$ partial $=0.063$ \\
\hline & Familiarity Position Congruency & $\mathrm{F} 1,113=5.17, p=0.025, \eta 2$ partial $=0.044$ \\
\hline & \multicolumn{2}{|r|}{ Between-Subject Effect } \\
\hline & Position & $\mathrm{F} 1,113=2.51, p=0.116, \eta 2$ partial $=0.022$ \\
\hline & Congruency & $\mathrm{F} 1,113=12.69, p=0.001, \eta 2$ partial $=0.101$ \\
\hline & Position Congruency & $\mathrm{F} 1,113=4.52, p=0.036, \eta 2$ partial $=0.038$ \\
\hline & Box test & $\mathrm{F} 3,608297=1.45, p=0.225$ \\
\hline & Levene test & Familiar: F3,113 $=25.95, p<0.001$ Unfamiliar: F3,113 $=2.82, p=0.042$ \\
\hline \multirow{11}{*}{ Recognition } & \multicolumn{2}{|r|}{ Within-Subject Effect } \\
\hline & Familiarity & $\mathrm{F} 1,113=5.92, p<0.017, \eta 2$ partial $=0.050$ \\
\hline & Familiarity Position & $\mathrm{F} 1,113=1.59, p=0.210, \eta 2$ partial $=0.014$ \\
\hline & Familiarity Congruency & $\mathrm{F} 1,113=0.29, p=0.594, \eta 2$ partial $=0.003$ \\
\hline & Familiarity Position Congruency & $\mathrm{F} 1,113=0.89, p=0.347, \eta 2$ partial $=0.008$ \\
\hline & \multicolumn{2}{|r|}{ Between-Subject Effect } \\
\hline & Position & $\mathrm{F} 1,113=3.23, p=0.075, \eta 2$ partial $=0.028$ \\
\hline & Congruency & $\mathrm{F} 1,113=22.21, p<0.001, \eta 2$ partial $=0.164$ \\
\hline & Position Congruency & $\mathrm{F} 1,113=5.04, p=0.027, \eta 2$ partial $=0.043$ \\
\hline & Box test & $\mathrm{F} 6,172097=4.68, p<0.001$ \\
\hline & Levene test & Familiar: F3,113 $=14.91, p<0.001$ Unfamiliar: F3,113 $=28.80, p=0.042$ \\
\hline
\end{tabular}




\subsection{Recall of Placed Brands}

In regard to the within-subject effects, triple interaction is seen between familiarity, position and congruence of the brand for recall (see Table 4), with a medium-low effect size $(\eta 2$ partial $=0.044)$. There is greater recall on the familiarity stimuli presented in the center and congruent, and less recall in the unfamiliar shown both in the center as and on the periphery and incongruent, as can be seen in Figure 2c,d. According to the Bonferroni test, statistically significant differences appear between the recall measurement for familiarity and congruency stimuli presented in the center and on the periphery, with greater recall for the first (Bonferroni $=0.26, p=0.004$ ), for the familiarity stimuli, and for those presented in the center between congruent and incongruent stimuli, with a higher median for the first (Bonferroni $=0.37, p<0.001$ ), and between the stimuli presented in the center and congruent between the familiar and unfamiliar, with a higher median for the first (Bonferroni test $=0.37, p<0.001$ ). This result does not confirm the hypothesis of the study, as it would be expected that recall would be greater for the familiar stimuli, presented in the center and incongruent. There is also interaction between familiarity and the congruency of the stimuli with the videogame's content, with a medium effect size $(\eta 2$ partial $=0.063)$. There are differences between the congruent and incongruent familiarity stimuli, with a higher mean for the congruent (Bonferroni $=0.22, p<0.001$ ) and differences between the familiar and unfamiliar congruent stimuli, with a higher mean for the familiar (Bonferroni $=0.22, p<0.001$ ) (see Figure $2 b$ ). There is no interaction between familiarity and positioning. In addition, there are statistically significant differences in the familiarity factor with a high effect size $(\eta 2$ partial $=0.143)$, with greater recall on the familiarity stimuli.

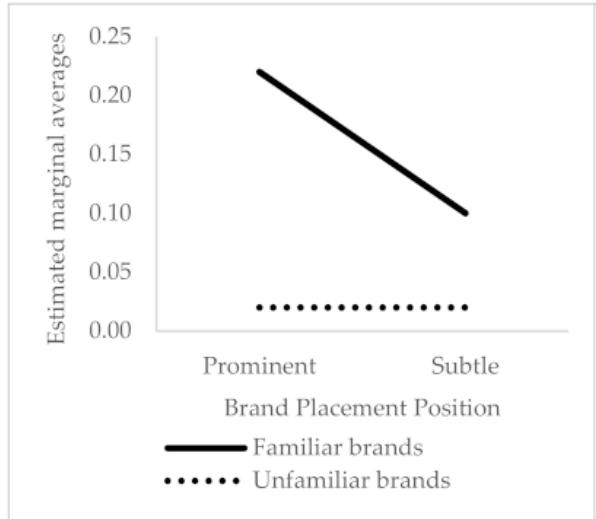

(a) Position $\times$ familiarity

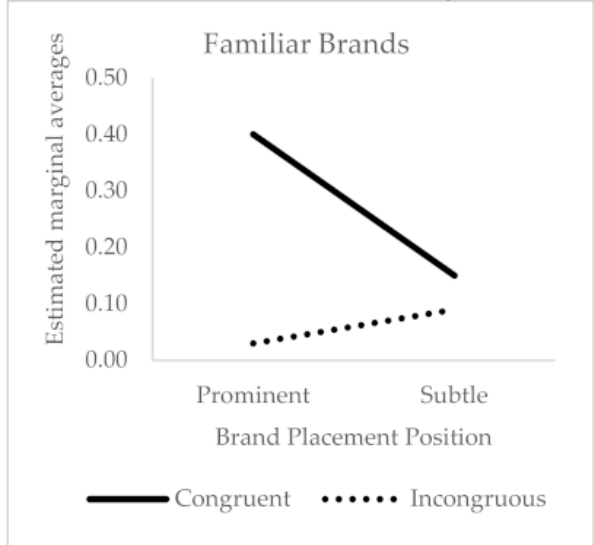

(c) For familiar brands, position $\times$ congruence

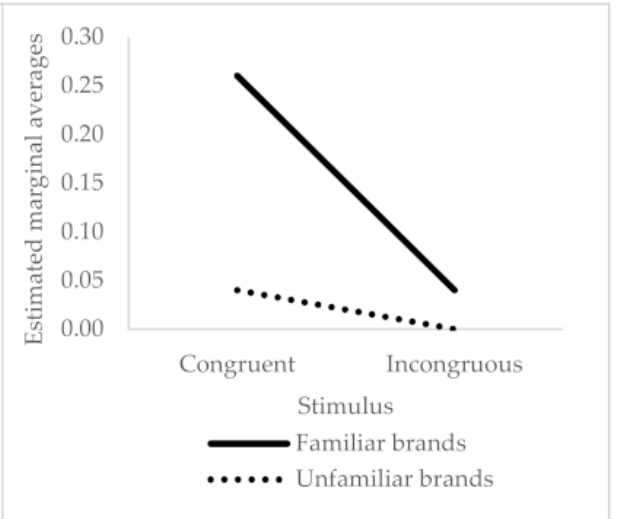

(b) Congruence $\times$ familiarity

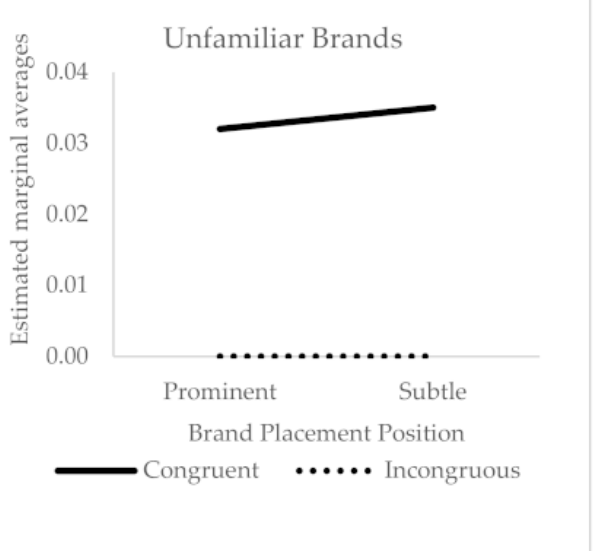

(d) For unfamiliar brands, position $\times$ congruence

Figure 2. Cont. 


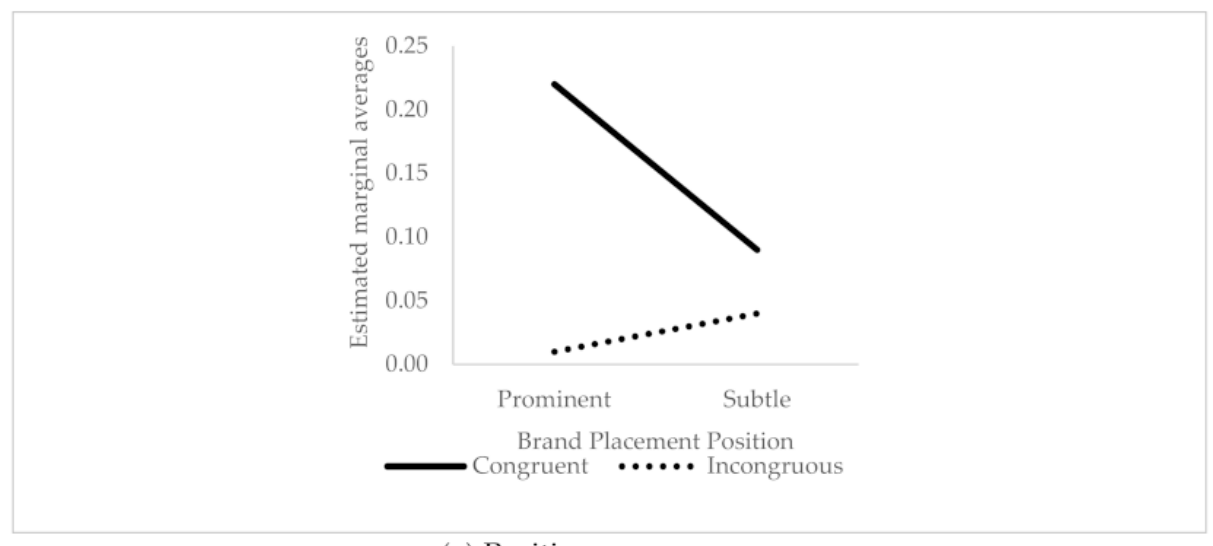

(e) Position $\times$ congruence

Figure 2. Recall measurements for each of the experimental conditions: (a) position $\times$ familiarity, (b) congruence $\times$ familiarity, (c) for familiar brands, position $\times$ congruence, $(d)$ for unfamiliar brands, position $\times$ congruence, $(\mathbf{e})$ position $\times$ congruence.

In regard to between-subject effects, interaction is seen between the position and congruency of the brand, with a medium-low effect size $(\eta 2$ partial $=0.038)$ (see Figure 2e). There are statistically significant differences for the congruent stimuli shown as prominent, which are recalled more than the subtle stimuli (Bonferroni $=0.13, p=0.010$ ), and the congruent stimuli shown as prominent are more remembered than the subtle (Bonferroni $=0.20, p<0.001$ ). In respect of the congruency factor, there are differences with a high effect size $(\eta 2$ partial $=0.101)$, with greater recall of the congruent stimuli. Finally, for the position factor, differences are not seen in recall.

\subsection{Recognition of Placed Brands}

The only within-subject factor where statistically significant differences appear is in familiarity, with a medium effect size $(\eta 2$ partial $=0.050)$ and greater recognition for the familiar brands. The hypothesis is not confirmed. Figure 3 shows the recognition measurements for the different conditions.

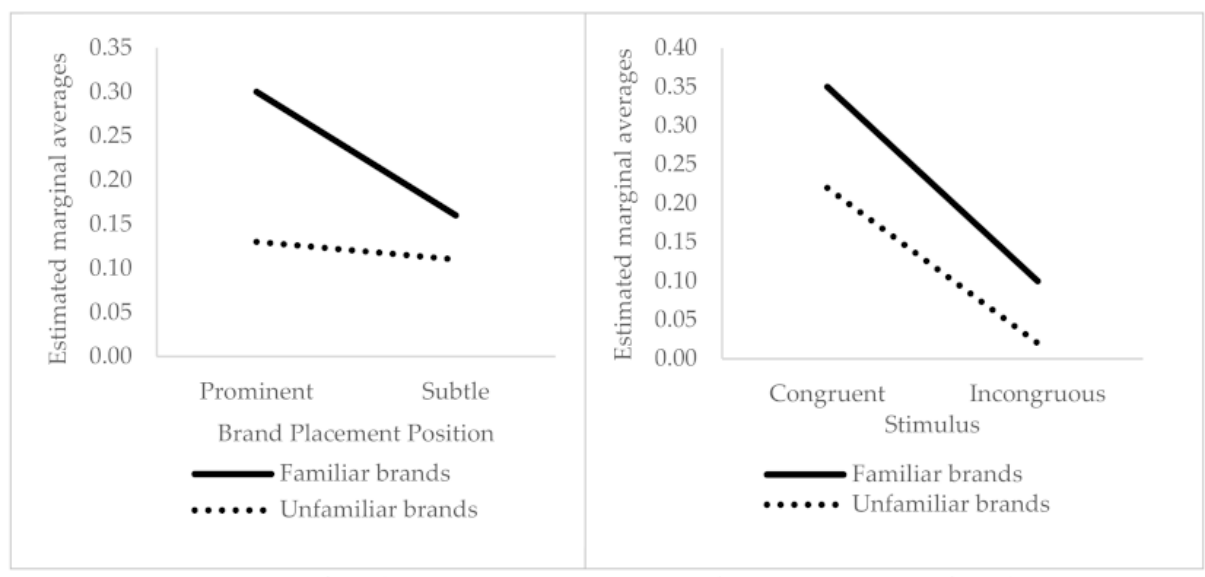

(a) Position $\times$ familiarity

(b) Congruence $\times$ familiarity

Figure 3. Cont. 


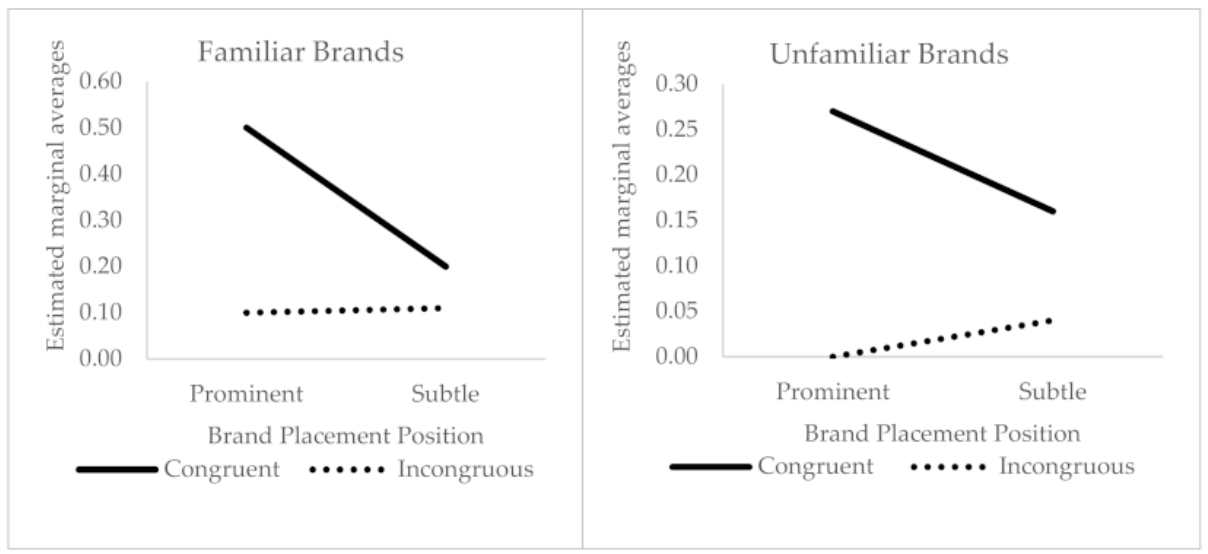

(c) For familiar brands, position $\times$ congruence

(d) For unfamiliar brands, position $\times$ congruence

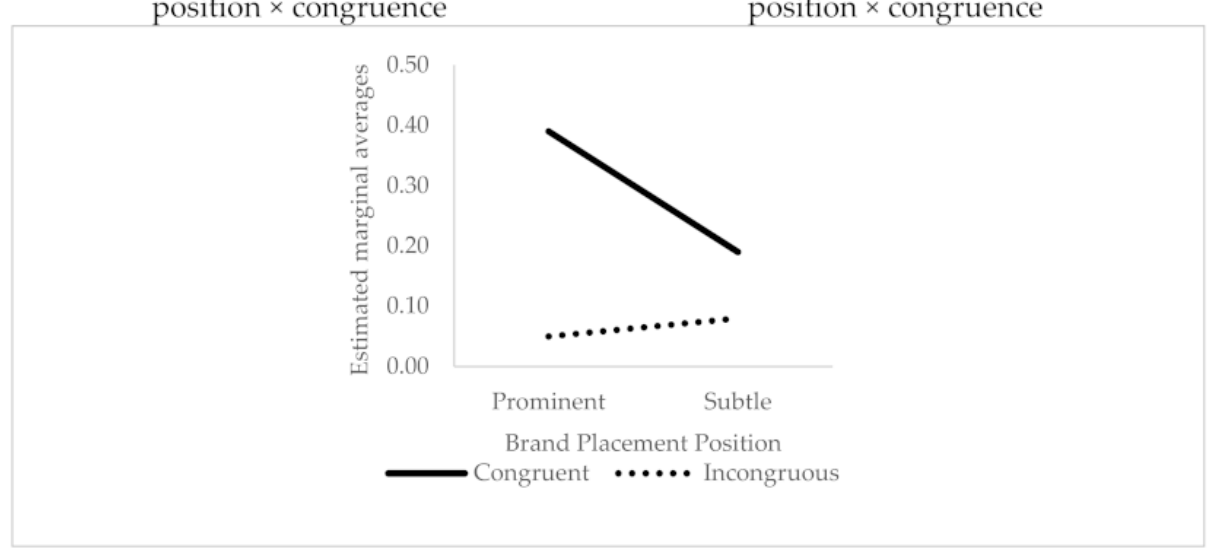

(e) Position $\times$ congruence

Figure 3. Recognition measurements for each of the experimental conditions: (a) position $\times$ familiarity, (b) congruence $\times$ familiarity, (c) for familiar brands, position $\times$ congruence, (d) for unfamiliar brands, position $\times$ congruence, $(\mathbf{e})$ position $\times$ congruence.

In regard to between-subject factors, there is interaction between position and congruency with a medium-low effect size $(\eta 2 p a r t i a l=0.043)$. Higher recognition is given for the congruent stimuli shown in the center as compared to those shown on the periphery (Bonferroni $=0.19, p=0.005$ ), and between the congruent stimuli shown in the center as compared with the incongruent (Bonferroni $=0.33, p<0.001$ ). There are statistically significant differences in the recognition in congruence and a high effect size ( $\eta 2$ partial $=0.164)$, with a higher mean for the congruent stimuli. Finally, differences in recognition based on position do not appear.

\section{Discussion and Conclusions}

Brand placement is a technique currently used in videogames, appearing as integrated into the background behind the action, which is processed passively and gives the subject realism and atmosphere [2]. As such, this technique seems a good way to promote brand recognition and its recall after being exposed to it while engaged in a leisure activity.

Within this area, research usually measures these variables separately $[25,26,39,40]$, combining them two by two $[27,35,41,50]$, or without any of the conditions included in this study, for example, measuring the effects in subtle positions [29,33]. For this reason, it was decided to explore the interaction of the three main variables that affect recall and recognition (position, familiarity and congruency), paying attention to all their possible combinations. In this context, this research has four implications related to memory.

First, the three main variables of BP presentation have been examined together to test how they influence the recall and recognition of the brands displayed. It was found that there was interaction. Apparently, the most effective way to recall and recognize 
a stimulus within a videogame on a racing circuit, and for the two market categories analyzed, automobiles and detergents, is by placing brands whose appearance in the game makes sense, are known to players who play this particular game, and are in the center of the screen. Having not found previous studies that analyze the interaction between all three, we believe this is a noteworthy result, despite the fact that the hypothesis on interaction is rejected, having discovered that the conjunction of the three-familiarity, a prominent position and congruency-promotes greater receptivity to the stimulus shown, which attracts more attention and, in turn, greater memorization.

Second, it provides an approach to the effects of brand placement on memory, based on whether brands are shown in the center of the screen or on the periphery. Nevertheless, the results show that position alone does not seem to have an influence, at least the position presented in this study, i.e., inflatables for prominent position, and banderoles for subtle position.

Third, it takes into account whether there are differences between processing an already familiar brand or one that is more novel. It is important to highlight that real brands have been shown, that is, ones that exist in the Spanish market. As such, it has been found that when one already has information stored about a brand shown in the videogame which is familiar, it is easier to bring it back in the memory, reflected in the recall and recognition tests $[25,26]$. This discovery is in line with what has been published in the literature and with our hypothesis $[15,25,26,34,35]$.

Fourth, it focuses on finding out if the congruency of the brand category is an influencing factor on whether some brands are committed to memory more than others. It was proposed that incongruent stimuli would attract more attention and be recalled and recognized better than congruent ones because of their novelty. Nevertheless, we obtained the opposite result, perhaps as the brands were consistent with the videogame played [42] or because cars are a market category that is more akin to the selected target than laundry detergents.

In addition, with respect to position, there was no interaction with familiarity, perhaps because the familiarity effect is so dominant that it does not matter if we show the brand in a prominent or subtle position. Indeed, congruency interacted with position, where placements congruent with the videogame and placed in a prominent position obtained better results, following the lines of prediction from the study by Peters and Leshner [24]. This discovery coincides with previous studies $[11,25,35]$ and with the spotlight model of attention [37]. Applying this to our study, it follows that, by having to drive a vehicle on a road, the spotlight is usually in the center part of the screen, which is where the road is. Similarly, there was interaction between the congruency and familiarity variables, with higher memorization when showing congruent and familiar stimuli. This is related to the assumption that, due to previous experiences, there is much more information on familiar brands, so it is easier to retrieve them from the memory, and even more so if these are aligned with the videogame $[25,26,34]$.

Consequently, the results of the present study reinforce that showing familiar stimuli $[26,39,40]$, or those located in a prominent position within a videogame $[11,15,35]$, are more noticed and remembered. Additionally, this helps in taking a position on the role of the congruence of brands placed in the chosen videogame, where brands that did indeed have a relationship (automobiles) showed better recall and recognition. As such, all of these findings support the idea of the relevance of studying brand placement.

\section{Main Implications}

Starting from the viewpoint that brand placement have a non-commercial appearance, this allows consumers to perceive brand placement as not being advertising, that is, not as advertisements [54]. Thus, including the brand and what it transmits is accepted as more credible, in contrast to a typical commercial message, where the aim is clear identification of the brand, giving it a leading role. That is why an in-game advertising type video game has been chosen. 
This article reflects the relevance of this assertion, highlighting the importance of taking into account the three variables addressed in the inclusion of BP in video games, where the main purpose is to increase their memorization. In particular, it seems that the most effective way to achieve greater memorization of the trademarks presented would be to put them in prominent positions as long as they were familiar to the target audience expected to play the game and related in some way to the subject of the video game, in this case, the driving or track racing genre. Therefore, these recommendations are relevant for a business when launching their brand strategy, whether to introduce a new brand or promote an existing one. Specifically, we consider that the first step a commercial brand should follow would be to analyze how familiar it is to the chosen segment of players. Following the findings of this research, if the brand is familiar to the target, it will have a better chance of being memorized, and even greater if it is congruent with the videogame genre. In the case of unfamiliarity, it would be convenient to investigate the types of video game genres and to use the brand placement technique in a congruent genre to increase the probability of memorization. Even so, it should be taken into account that it is necessary to delve further into how these factors help to increase the memorization of the inserted BP depending on the videogame in which it is presented. It is affirmed that video games are here to stay and are becoming more and more popular among the population.

\section{Limitations and Future Research}

This research had some limitations that it is desirable to reduce in future studies. In respect to the videogame, we only tested one genre, a racing game where players race against the clock. There is a wide variety of videogame genres in terms of roles and how they are played, so future research should use other videogame genres, such as sports or shooting games, to see if the results found can be extrapolated. Additionally, we believe that combining other variables, such as attitude or immersion in the videogame, can have an influence on memorization of brand placement.

Furthermore, another limitation was the inability to change the order in which the brands were presented within each game, as the game platform did not allow this. Nor was it possible to create more groups where the brands were reversed, based on whether the brand was familiar or not. Therefore, the familiar brand was always seen before the unfamiliar one. Besides, although it is true that the entire sample played video games, only students were included. In any case, the age range coincides with real consumers who spend the most time playing video games [48]. As such, in the future it would be advisable to include other representative samples of the general population in order to generalize the results found.

Finally, we propose delving further into the area of implicit memory $[45,50,55]$ and try another longitudinal phase to see if memory and recognition change over time. On the one hand, this could involve using eye movement measurements aimed at confirming that players look at brand placement and for how long, that is, obtaining attentional data. Likewise, use of an electroencephalogram to measure if there is codification of the brands would be interesting, to see if there is a correlation with stated recall and recognition measurements. Moreover, physiological measurements, such as heart rate or sweating, may shed light on what the impact is or on the activation that occurs when paying attention to brand placement.

Author Contributions: Conceptualization, I.A., J.P. and M.D.M.; Methodology, I.A., J.P. and M.D.M.; Software, I.A., J.P. and M.D.M.; Validation, I.A., J.P. and M.D.M.; Formal Analysis, I.A., J.P. and M.D.M.; Investigation, I.A., J.P. and M.D.M.; Resources, I.A., J.P. and M.D.M.; Data Curation, I.A., J.P. and M.D.M.; Writing—Original Draft Preparation, I.A., J.P. and M.D.M.; Writing—Review \& Editing, I.A., J.P. and M.D.M.; Visualization, I.A., J.P. and M.D.M.; Supervision, I.A., J.P. and M.D.M.; Project Administration, I.A., J.P. and M.D.M. All authors have read and agreed to the published version of the manuscript.

Funding: This research received no external funding. 
Institutional Review Board Statement: The study was conducted according to the guidelines of the Declaration of Helsinki, and approved by the Ethics Committee at Centro de Enseñanza Superior Cardinal Cisneros (22 November 2018), affiliated with the Universidad Complutense de Madrid, adhering to the standard ethical protocols from the World Medical Association Declaration of Helsinki in 1964. Participants were informed about their right to withdraw from the study without any repercussions and they signed the informed consent prior to participation.

Informed Consent Statement: Informed consent was obtained from all subjects involved in the study.

Data Availability Statement: The data presented in this study are available on request from the corresponding author. The data are not publicly available due to privacy and ethical restrictions.

Acknowledgments: This research has been greatly assisted by Luis Caballero, who edited the game from the Unreal Engine Platform (https:/ / www.unrealengine.com).

Conflicts of Interest: The authors declare no conflict of interest.

\section{References}

1. Chaney, I.; Hosany, S.; Wu, M.-S.; Chen, C.-H.S.; Nguyen, B. Size does matter: Effects of in-game advertising stimuli on brand recall and brand recognition. Comput. Hum. Behav. 2018, 86, 311-318. [CrossRef]

2. Chang, Y.; Yan, J.; Zhang, J.; Luo, J. Online In-Game Advertising Effect: Examining the Influence of a Match Between Games and Advertising. J. Interact. Advert. 2010, 11, 63-73. [CrossRef]

3. Lord, K.R.; Gupta, P.B. Response of buying-center participants to B2B product placements. J. Bus. Ind. Mark. 2010, 25, 188-195. [CrossRef]

4. Redker, C.; Gibson, B.; Zimmerman, I. Liking of Movie Genre Alters the Effectiveness of Background Product Placements. Basic Appl. Soc. Psychol. 2013, 35, 249-255. [CrossRef]

5. Cowley, E.; Barron, C. When Product Placement Goes Wrong: The Effects of Program Liking and Placement Prominence. J. Advert. 2008, 37, 89-98. [CrossRef]

6. Van Reijmersdal, E.A.; Boerman, S.C.; Buijzen, M.; Rozendaal, E. This is Advertising! Effects of Disclosing Television Brand Placement on Adolescents. J. Youth Adolesc. 2017, 46, 328-342. [CrossRef]

7. Delattre, E.; Colovic, A. Memory and perception of brand mentions and placement of brands in songs. Int. J. Advert. 2009, 28, 807-842. [CrossRef]

8. Ferguson, N.S.; Burkhalter, J.N.; Yo, D.J. That's My Brand: An Examination of Consumer Response to Brand Placements in Hip-Hop Music. J. Advert. 2014, 44, 47-57. [CrossRef]

9. Lai, Y.; Lai, C.; Chiang, H. Exploring the effects of online video advertising message display, product placement, and product involvement on advertising effectiveness. Int. J. Econ. Stat. 2015, 3, 117-127.

10. Dagnino, G. Regulation and co-regulation of product placement for OTT SVODs: The case of Netflix. Int. J. Digit. Telev. 2018, 9, 203-218. [CrossRef]

11. Nelson, M.R. Recall of Brand Placements in Computer/Video Games. J. Advert. Res. 2002, 42, 80-92. [CrossRef]

12. Schneider, L.-P.; Systems, B.; Cornwell, T.B. Cashing in on crashes via brand placement in computer games. Int. J. Advert. 2005, 24, 321-343. [CrossRef]

13. Newzoo Global Games Market Report. Available online: https://resources.newzoo.com/hubfs/Reports/Newzoo_2018_Global_ Games_Market_Report_Light.pdf?submissionGuid=9fd6867b-4cd9-433c-b8fb-97bb9ca64f78 (accessed on 1 December 2020).

14. Yang, M.; Roskos-Ewoldsen, D.R.; Dinu, L.; Arpan, L.M. The Effectiveness of "in-Game" Advertising: Comparing College Students' Explicit and Implicit Memory for Brand Names. J. Advert. 2006, 35, 143-152. [CrossRef]

15. Mau, G.; Silberer, G.; Constien, C. Communicating brands playfully: Effects of in-game advertising for familiar and unfamiliar brands. Int. J. Advert. 2008, 27, 827-851. [CrossRef]

16. Karisik, V.J. 20 Years of Research on Product Placement in Movie, Television and Video Game Media. J. Econ. Soc. Stud. 2014, 4, 98-108.

17. Wang, Y.; Yao, M.Z. Did You Notice the Ads? Examining the Influence of Telepresence and User Control on the Effectiveness of Embedded Billboard Ads in a VR Racing Game. J. Interact. Advert. 2020, 20, 258-272. [CrossRef]

18. Balasubramanian, S.K.; Karrh, J.A.; Patwardhan, H. Audience Response to Product Placements: An Integrative Framework and Future Research Agenda. J. Advert. 2006, 35, 115-141. [CrossRef]

19. Cholinski, A. The Effectiveness of Product Placement: A Field Quasi-experiment. Int. J. Mark. Stud. 2012, 4, 14-28. [CrossRef]

20. Hang, H.; Auty, S. Children playing branded video games: The impact of interactivity on product placement effectiveness. $J$. Consum. Psychol. 2011, 21, 65-72. [CrossRef]

21. Van Reijmersdal, E.A.; Jansz, J.; Peters, O.; Van Noort, G. The effects of interactive brand placements in online games on children's cognitive, affective, and conative brand responses. Comput. Hum. Behav. 2010, 26, 1787-1794. [CrossRef]

22. Martí-Parreño, J. Marketing y videojuegos. Product Placement, in-Game Advertising y Advergaming; ESIC Editorial: Madrid, Spain, 2010. 
23. Lee, M.; Faber, R.J. Effects of Product Placement in On-Line Games on Brand Memory: A Perspective of the Limited-Capacity Model of Attention. J. Advert. 2007, 36, 75-90. [CrossRef]

24. Peters, S.; Leshner, G. Get in the Game: The Effects of Game-Product Congruity and Product Placement Proximity on Game Players' Processing of Brands Embedded in Advergames. J. Advert. 2013, 42, 113-130. [CrossRef]

25. Hwang, Y.; Ballouli, K.; So, K.; Heere, B. Effects of Brand Congruity and Game Difficulty on Gamers' Response to Advertising in Sport Video Games. J. Sport Manag. 2017, 31, 480-496. [CrossRef]

26. Martí-Parreño, J.; Bermejo-Berros, J.; Aldás-Manzano, J. Product Placement in Video Games: The Effect of Brand Familiarity and Repetition on Consumers' Memory. J. Interact. Mark. 2017, 38, 55-63. [CrossRef]

27. Kim, E.; Eastin, M.S. External Brand Placement: The Effects on Game Players' Processing of an In-Game Brand. J. Promot. Manag. 2015, 21, 391-411. [CrossRef]

28. Schacter, D.L. Implicit memory: History and current status. J. Exp. Psychol. Learn. Mem. Cogn. 1987, 13, 501-518. [CrossRef]

29. Lull, R.B.; Gibson, B.; Cruz, C.; Bushman, B.J. Killing characters in video games kills memory for in-game ads. Psychol. Popul. Media Cult. 2018, 7, 87-97. [CrossRef]

30. Srinivas, K.; Roediger, H.L. Classifying implicit memory tests: Category association and anagram solution. J. Mem. Lang. 1990, 29, 389-412. [CrossRef]

31. Kintsch, W. Models for Free Recall and Recognition. In Models of Human Memory; Elsevier BV: Amsterdam, The Netherlands, 1970; pp. 331-373.

32. Egeth, H.; Kahneman, D. Attention and Effort. Am. J. Psychol. 1975, 88, 339. [CrossRef]

33. Chaney, I.M.; Lin, K.-H.; Chaney, J. The Effect of Billboards within the Gaming Environment. J. Interact. Advert. 2004, 5, 37-45. [CrossRef]

34. Gangadharbatla, H. A comparison of in-game brand placement for active versus passive players. J. Interact. Advert. 2016, 16, 117-132. [CrossRef]

35. Jeong, E.J.; Biocca, F.A. Are there optimal levels of arousal to memory? Effects of arousal, centrality, and familiarity on brand memory in video games. Comput. Hum. Behav. 2012, 28, 285-291. [CrossRef]

36. Williams, R.B. Location, Integration, Interruption: Visual Properties and Recognition of Video Game Advertising. J. Promot. Manag. 2020, 26, 253-276. [CrossRef]

37. Eriksen, C.W.; Yeh, Y.-Y. Allocation of Attention in the Visual Field. J. Exp. Psychol. Hum. Percept. Perform. 1985, 11, 583-597. [CrossRef]

38. Law, S.; Braun, K.A. I'll Have What She's Having: Gauging the Impact of Product Placements on. Psychol. Mark. 2000, 17, 1059-1075. [CrossRef]

39. Choi, Y.K.; Lee, S.M.; Li, H. Audio and Visual Distractions and Implicit Brand Memory: A Study of Video Game Players. J. Advert. 2013, 42, 219-227. [CrossRef]

40. Siemens, J.C.; Smith, S.; Fisher, D. Investigating the Effects of Active Control on Brand Recall Within In-Game Advertising. J. Interact. Advert. 2015, 15, 43-53. [CrossRef]

41. Vashisht, D.; Sreejesh, S.P. Are they really persuaded with the brand embedded in the game? Analyzing the effects of nature of game, brand prominence and game-product congruence. J. Res. Interact. Mark. 2016, 10, 1-20.

42. Lewis, B.; Porter, L. In-Game Advertising Effects. J. Interact. Advert. 2010, 10, 46-60. [CrossRef]

43. Gross, M.L. Advergames and the effects of game-product congruity. Comput. Hum. Behav. 2010, 26, 1259-1265. [CrossRef]

44. Dahlén, M.; Rosengren, S.; Törn, F.; Öhman, N. Could Placing ADS Wrong be Right? Advertising Effects of Thematic Incongruence. J. Advert. 2008, 37, 57-67. [CrossRef]

45. Ghosh, T. Winning Versus Not Losing: Exploring the Effects of In-Game Advertising Outcome on its Effectiveness. J. Interact. Mark. 2016, 36, 134-147. [CrossRef]

46. Machleit, K.A.; Allen, C.T.; Madden, T.J. The Mature Brand and Brand Interest: An Alternative Consequence of Ad-Evoked Affect. J. Mark. 1993, 57, 72-82. [CrossRef]

47. Bosshard, S.S.; Bourke, J.D.; Kunaharan, S.; Koller, M.; Walla, P.; Heinonen, J. Established liked versus disliked brands: Brain activity, implicit associations and explicit responses. Cogent Psychol. 2016, 3, 1-30. [CrossRef]

48. Entertainment Software Association. Essential Facts About the Computer and Video Game Industry. Available online: https:// www.theesa.com/wp-content/uploads/2019/05/2019-Essential-Facts-About-the-Computer-and-Video-Game-Industry.pdf (accessed on 1 December 2020).

49. Gangadharbatla, H.; Bradley, S.; Wise, W. Psychophysiological Responses to Background Brand Placements in Video Games. J. Advert. 2013, 42, 251-263. [CrossRef]

50. Vermeir, I.; Kazakova, S.; Tessitore, T.; Cauberghe, V.; Slabbinck, H. Impact of flow on recognition of and attitudes towards in-game brand placements: Brand congruence and placement prominence as moderators. Int. J. Advert. 2014, 33, 785-810. [CrossRef]

51. Cohen, J. A Power Primer. Psychol. Bull. 1992, 112, 155-159. [CrossRef]

52. Asociación Española de Videojuegos. La Industria del Videojuego en España. Anuario. 2019. Available online: http://www.aevi. org.es/web/wp-content/uploads/2020/04/AEVI-ANUARIO-2019.pdf (accessed on 22 January 2021).

53. Pardo, A.; San-Martín, R. Análisis de Datos en Ciencias Sociales y de La Salud, Volumen II; Síntesis: Madrid, Spain, 2010. 
54. Gillespie, B.; Joireman, J. The Role of Consumer Narrative Enjoyment and Persuasion Awareness in Product Placement Advertising. Am. Behav. Sci. 2016, 60, 1510-1528. [CrossRef]

55. D'Hooge, S.C.; Hudders, L.; Cauberghe, V. Direct evaluative conditioning in brand placement: The impact of scene valence and prominence on brand placement repetition effects. J. Consum. Behav. 2017, 16, 452-462. [CrossRef] 